\title{
The Nesfatin-1 Pathway from Hippocampus to Ventromedial Nucleus and its Regulation on Gastric Motility in Diabetic Rats
}

\author{
Baohua Jing ${ }^{1}$, Qing Yang ${ }^{2}$, Weidong Xin ${ }^{2}$, Luo $\mathrm{Xu}^{1}$
}

${ }^{1}$ Dept. of Pathophysiology, Medical College of Qingdao University, Qingdao, Shandong, 266021, China; 2 JIAOZHOU People's Hospital, Jiaozhou, Shandong, 266300, China

\begin{abstract}
Objective: To investigate the effect of microinjection of Nesfatin-1 into VMH on gastric motility in diabetic rats and its regulatory mechanism, and to study whether electrical stimulation of hippocampal CA1 region can regulate this process. Methods: The diabetic rat model was established, and the hippocampal-VMH nesfatin-1 pathway was observed by retrograde gold fluorescence tracing combined with fluorescence immunohistochemistry. The effects of microinjection of nesfatin-1 by VMH and electrical stimulation of hippocampus on gastric motility were observed in vivo. Results: Nesfatin-1 inhibited gastric motility in a dose-dependent manner, and astressin-B partially blocked the inhibition of nesfatin-1 on gastric motility; Nesfatin-1 immunoreactive neurons were present in the cytoplasm of hippocampal CA1 region and Nesfatin-1 was expressed in some fluorescent gold labeled cells; electrostimulation of hippocampal CA1 region could promote gastric motility in diabetic rats, and anti-NUCB2/Nesfatin-1 antibody could promote gastric motility in diabetic rats.Enhance the effect of electrical stimulation of hippocampal CA1 region on gastric motility. Conclusion: Nesfatin-1 injection into VMH can regulate gastric motility in diabetic rats, which may be related to the CRF system, and the hippocampal CA1 region participates in the regulation of gastric motility by nesfatin-1 in VMH.
\end{abstract}

Keywords: Hi, VMH, diabetic Rats, Gastric Motility

\begin{abstract}
Introduction
Diabetes mellitus is a metabolic disease with elevated blood sugar. Without standard treatment, long-term hyperglycemia can lead to various complications. Diabetic gastroparesis is a common complication of diabetes mellitus, and the incidence is increasing year by year. The pathogenesis of diabetic gastroparesis is very complex, and it is still unclear. Studies have shown that the occurrence of diabetic gastroparesis is related to many factors, including hyperglycemia, genetic factors, autonomic neuropathy and so on. The mechanism of diabetic gastroparesis is mostly focused on peripheral gastrointestinal hormone secretion, smooth muscle degeneration and so on, while the central mechanism of diabetic gastroparesis is rarely reported.
\end{abstract}

Nesfatin-1, an endocrine peptide involved in appetite regulation, is a cleavage product of nuclear histone 2 (NUCB2), which contains 82 amino acids ${ }^{[1]}$. Nesfatin-1 was first found in the hypothalamus of rats by O-I et al. Later studies showed that Nesfatin-1 could play a variety of roles in the central and peripheral regions.Nesfatin-1 is widely distributed in feeding-related brain regions, such as paraventricular nucleus (PVN), arcuate nucleus (ARC), ventromedial nucleus (VMH), hippocampus (Hi), amygdala, septal nucleus, brainstem, dorsal vagal nucleus, solitary tract nucleus and posterior region ${ }^{[2}$. It has been reported that Nesfatin-1 inhibits nocturnal feeding and gastric motility in mice, and dose-dependent inhibition of gastric emptying and gastric acid secretion in rats by central injection of Nesfatin- ${ }^{[3-5]}$. In addition to appetite regulation, Nesfatin-1 is involved in many pathophysiological processes such as stress, reproduction, depression and anxiety.

Hippocampus is a part of the marginal nervous system. It is mainly involved in the functional regulation of learning and memory. There are extensive fibrous connections between hippocampus and hypothalamus, brainstem and other regional centers. It jointly regulates visceral functional activities, also known as "visceral brain" ${ }^{[6]}$. In recent years, more and more attention has been paid to the study of hippocampus in feeding and digestion ${ }^{[7]}$. VMH is also an important brain area involved in food intake. Studies have found that VMH lesions can lead to increased food intake and obesity, so VMH is known as the "satiety center" of the brain $^{[8]}$. The effect of Nesfatin-1 in VMH on gastric motility in rats has not been reported yet.

Therefore, in this study, we mainly observed the composition of nerve pathway in VMH-hippocampal 
CA1 region, explored the effect of microinjection of Nesfatin-1 into VMH on gastric motility in diabetic rats and its regulatory mechanism, and studied whether electrical stimulation of hippocampal CA1 region could regulate this process.

\section{Materials and Methods \\ 1.1 Animals}

Male Wistar rats, weighing 250-300 g, were fed at room temperature $\left(25 \pm 2^{\circ} \mathrm{C}\right)$ and circadian circulating light (08:00-20:00). All rats were fed with standard laboratory diet, free eating and drinking water. All animal experiments are strictly carried out in accordance with the "Measures for the Protection and Use of Laboratory Animals of Qingdao University"

\subsection{Establishment of diabetic rat model}

120 rats were selected to establish diabetic rat model. The rats were fasting for 12 hours before the start of the experiment and could not stop drinking water. Rats in model group were intraperitoneally injected with 35 $\mathrm{mg} / \mathrm{kg} 1 \%$ streptozotocin (STZ) solution for one week after the first injection and the same dose of $35 \mathrm{mg} / \mathrm{kg}$ $1 \%$ STZ solution for the second intraperitoneal injection on the eighth day. The fasting blood glucose of rats was measured by tail tip blood. In the experiment, 96 diabetic rats were selected as diabetic models with fasting blood glucose $(\geq 7.8 \mathrm{mmol} / \mathrm{l})$ and postprandial blood glucose $(\geq 11.1 \mathrm{mmol} / \mathrm{l})$.

1.3 Fluorescent gold retrograde tracing and fluorescent immunohistochemical experiments

Eight diabetic rats were randomly selected. After $10 \%$ chloral hydrate anesthesia $(0.3 \mathrm{ml} / 100 \mathrm{~g})$, they were fixed in a stereotaxic brain locator to expose the skull. VMH (2.28-2.64 $\mathrm{mm}$ before and after the fontanel, 0.1-1.2 mm beside and 9.0-10.0 mm deep) was drilled at the VMH site according to the brain atlas of Paxinos $\&$ Watson rats. The fluorescent gold of $0.15 \mathrm{~L} 3 \%(\mathrm{w} / \mathrm{v})$ was extracted by microinjector and injected into $\mathrm{VMH}$ slowly for one minute to make the fluorescent gold fully diffuse. Suture scalp incision of rats, intraperitoneal injection of 80,000 units of penicillin for 3 days after operation to prevent postoperative infection. Rats were placed in a separate cage. After 7 days of recovery, intraperitoneal injection of $10 \%$ chloral hydrate $(0.3 \mathrm{ml} / 100 \mathrm{~g})$ was fixed on the operating table. $200 \mathrm{ml} 0.9 \%$ saline and $200 \mathrm{ml} 4 \%$ polyformaldehyde were injected successively for perfusion and fixation. Rats were decapitated and their brains were taken out. Frozen section machine continuous coronal section, slice thickness $15 \mathrm{~m}$, all slices placed in - $20 \mathrm{C}$ refrigerator to avoid light and freeze. The appropriate brain sections were selected for immunohistochemical experiments.

Brain slices were washed with normal saline and 0.01 mol PBS solution for 3 times x 5 minutes. Rat anti-NUCB2/Nesfatin-1 $\operatorname{lgG}$ antibody was added, 4_C overnight, $0.01 \mathrm{~mol}$ PBS solution was washed three times for x 5 minutes.Cy3-labeled sheep anti-mouse $\operatorname{lgG}$ antibody was added and incubated at room temperature for 2 hours. Wash again with $0.01 \mathrm{~mol}$ PBS solution for 3 times x 5 minutes. The results were photographed with BX50 microscope and DP50 digital camera after quenching-proof fluorescent sealing tablet.

\subsection{In vivo gastric exercise experiment}

Forty diabetic rats were randomly divided into four groups ( $\mathrm{n}=10)$ : (1) $0.08 \mathrm{~g} / 0.5 \mathrm{~L}$ Nesfatin-1 group; (2) $0.8 \mathrm{~g} / 0.5$ L Nesfatin-1 group; (3) $8.0 \mathrm{~g} / 0.5$ L Nesfatin-1 group; (4) 0.5 L NS group.

The rats were anesthetized with $10 \%$ chloral hydrate $(0.3 \mathrm{ml} / 100 \mathrm{~g})$, fixed on the operating table, opened the abdominal cavity, and fully exposed the gastric tissue. At the position of $0.5 \mathrm{~cm}$ from the pylorus of stomach, stress sensors and bipolar silver wire electrodes were attached to the ventral and dorsal serosal surfaces of the antrum along the direction of the gastric annular muscle. The wires were subcutaneously directed to the neck of the rat and sutured through the abdominal incision. The animals were given normal diet for 3 days after operation. After operation, rats were fed in separate cages and intraperitoneally injected with 80,000 units of penicillin every day to prevent infection.[9]

Before recording gastric motility, the rats were fasted for 18 hours and placed in a special cage. When the animals were awake, the stress sensor was connected with a bridge amplifier to record the amplitude and frequency of gastric contraction in rats. The gastric contraction movement was recorded for 30 minutes before and 60 minutes after administration. The frequency and amplitude of gastric contraction were observed before and after administration, and the change rate was calculated. Each rat was recorded 1-2 hours a day for at least 2 days.

\subsection{Electrical stimulation}

48 diabetic rats were randomly divided into 6 groups $(\mathrm{n}=8)$ : SS group; ES group; NR+SS group; NR+ES group; anti-Nn-Ab+SS group; anti-Nn-Ab+ES group.

Unipolar stimulus electrodes were implanted into hippocampal CA1 region to induce electrical stimulation. According to the rat brain atlas, the unipolar stimulator electrodes were inserted vertically into the hippocampal CA1 region (3.14-3.6 $\mathrm{mm}$ after the anterior fontanel, $1.3-2.0 \mathrm{~mm}$ beside the fontanel, 2.6-3.2 mm deep, Fig. 5). The stimulator was stimulated by high frequency square wave current pulse $(20 \mathrm{~A}$, lasting $0.5 \mathrm{~ms}$ ). All surgical procedures were the same in the pseudo-stimulation group and the stimulation group, but no current was passed through the stimulation electrode.The gastric motility of conscious rats in body position was recorded for 60 minutes.

\subsection{Statistical analysis}

SPSS 18.0 and PPMS 1.5 software were used to analyze the data. All the data were analyzed as follows: 
Means of multiple samples were compared by one-way ANOVA. Means of samples between the two groups were compared by $t$ test, $\mathrm{P}<0.05$ showed significant difference.

\section{Result}

2.1 Effect of intravenous injection of Nesfatin-1 in 1 VMH on gastric motility in diabetic rats

Rats were injected with 0.5 L Nesfatin-1 (0.08, $0.8,8.0$ g) at different concentrations of VMH. The amplitude and frequency of gastric contraction in rats were significantly reduced and showed dose-effect dependence ( $\mathrm{P}<0.05-0.01$, Fig. 1). Compared with the $0.8 \mathrm{~g} / 0.5 \mathrm{~L}$ Nesfatin-1 group, the amplitude and frequency of gastric contraction increased significantly after intravenous injection of $0.5 \quad \mathrm{~L} \quad(0.8 \mathrm{~g}$ Nesfatin-1+30 $\mathrm{g}$ astressin-B) in VMH $(\mathrm{P}<0.05$, Fig. 1).There was no significant change in the amplitude and frequency of gastric contraction in rats treated with astressin-B or saline alone ( $\mathrm{P}>0.05$, Fig. 1$)$.
A

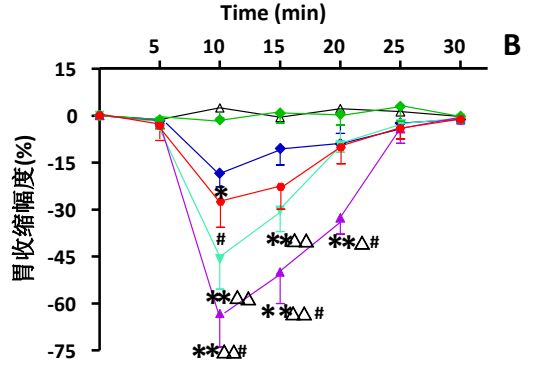

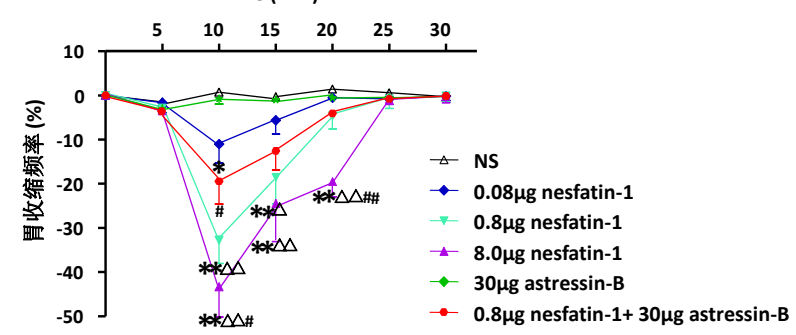

Fig. 1 Effect of Nesfatin-1 injection into ventromedial hypothalamic nucleus on gastric motility in diabetic rats

A: The effect of Nesfatin-1 in ventromedial hypothalamic nucleus on gastric contraction in diabetic rats; B: The effect of Nesfatin-1 in ventromedial hypothalamic nucleus on gastric contraction frequency in diabetic rats

$* \mathrm{P}<0.05$, ${ }^{* *} \mathrm{P}<0.01$, as compared with NS group; $\triangle \mathrm{P}<0.05, \Delta \triangle \mathrm{P}<0.01$, as compared with $0.08 \mathrm{~g}$ Nesfatin-1; ${ }^{*} \mathrm{P}$ $<0.05,{ }^{\# \#} \mathrm{P}<0.01$, as compared with $0.8 \mathrm{~g}$ Nesfatin-1 group

\section{2 Expression of FG/Nesfatin-1 double labeled} neurons in hippocampal CA1 region

In retrograde Fluorogold tracing experiment, ratsAfter injecting $0.1 \mathrm{~L} 3 \%$ fluorescent gold into $\mathrm{VMH}$, fluorescent gold labeled neurons were found in hippocampal CA1 area 7 days later (Fig. 2).Fluorescence immunohistochemical staining with
Nesfatin-1 antibody at the same level showed that Nesfatin-1 immunoreactive neurons were present in the cytoplasm of hippocampal CA1 region (Fig. 2), and Nesfatin-1 was expressed in some fluorescent gold labeled cells (Fig. 2), suggesting that Nesfatin-1 neurons in hippocampal CA1 region could project fibers to VMH.
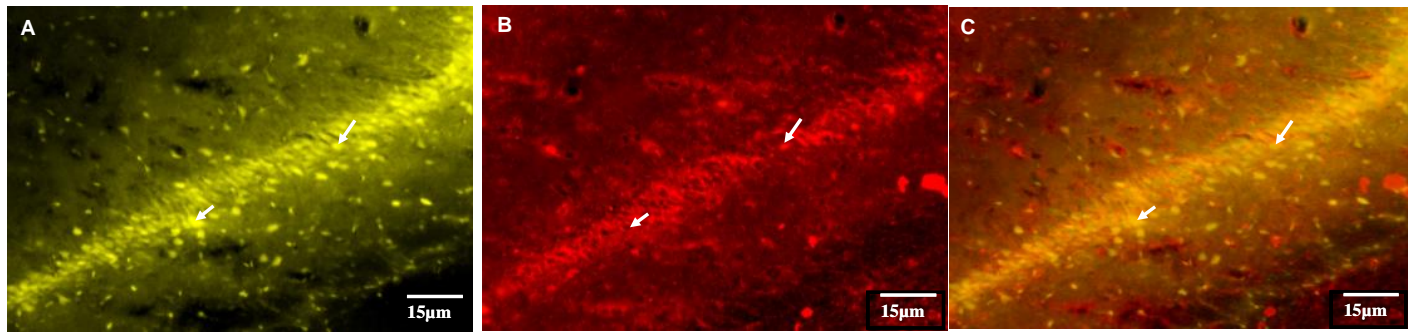

Fig. 2 FG/Nesfatin-1 neurons in hippocampal CA1 region of diabetic rats A: FG-labeled neurons in hippocampal CA1 region; B: Nesfatin-1 immunopositive neurons in hippocampal CA1 region; C: FG/Nesfatin-1 double labeled neurons in hippocampal CA1 region 2.2 In vivo studies

\section{3 Effect of electric stimulation of Hi CA1 region on gastric motion in diabetic rats}

The results showed that the amplitude and frequency of gastric contraction in diabetic rats were significantly increased by electrical stimulation of hippocampal CA1 ( $\mathrm{P}<0.05-0.01$, Fig. 3). Compared with the normal sheep serum plus electrical stimulation group, the amplitude and frequency of gastric contraction in rats were further enhanced by pre-injection of anti-NUCB2/Nesfatin-1 antibody into VMH before electrical stimulation of hippocampal CA1.Compared with anti-NUCB2/Nesfatin-1 antibody plus pseudoelectric stimulation group, the gastric contraction amplitude and frequency of anti-NUCB2/Nesfatin-1 antibody plus electric stimulation group were significantly increased $(\mathrm{P}<0.05$, figure 3).Normal sheep serum or anti-NUCB2/Nesfatin-1 antibody in rat VMH had no significant effect on gastric motility (P > 0.05, Fig. 3). 
A

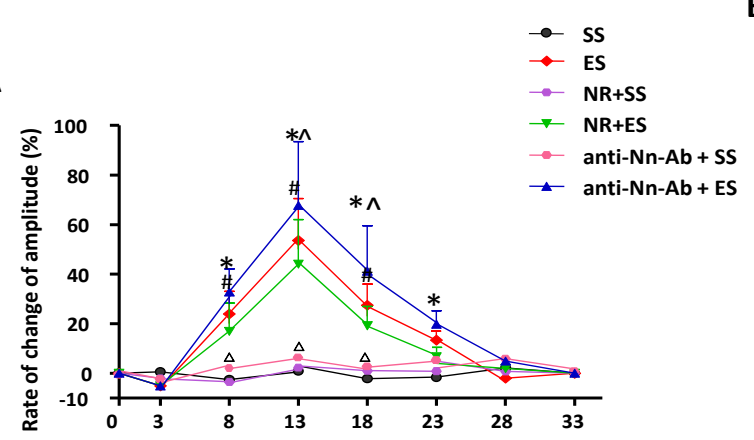

B

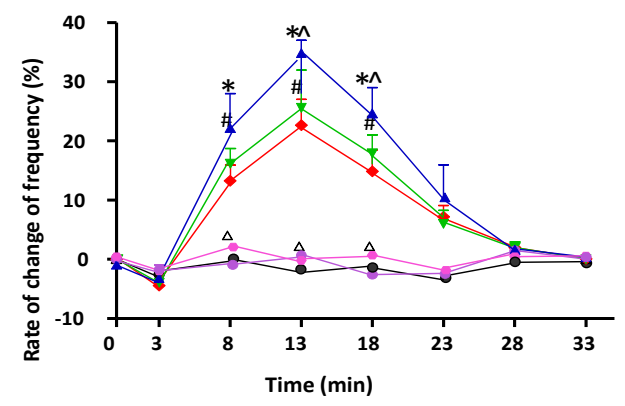

Fig. 3 Effects of electrical stimulation of hippocampal CA1 region on gastric motility in diabetic rats

A: The effect of electrical stimulation of hippocampal CA1 region on gastric contraction amplitude in rats; B: The effect of electrical stimulation of hippocampal CA1 region on gastric contraction frequency in rats

${ }^{\#} \mathrm{P}<0.01$ vs. SS group; $\triangle \mathrm{P}<0.01$ vs vs. NR+SS group; $* \mathrm{P}<0.05$ vs. anti-Nn-Ab+SS group; ${ }^{\wedge} \mathrm{P}<0.01$ vs. anti-Nn-Ab+SS group; ${ }^{\wedge} \mathrm{P}<0.05$ vs. $\mathrm{NR}+\mathrm{ES}+\mathrm{SS}$ group

\section{Discussion}

The results of this study showed that administration of Nesfatin-1 in VMH of diabetic rats decreased gastric motility in a dose-dependent manner, and astressin-B, an adrenocorticotropic receptor antagonist, partially neutralized the effect of Nesfatin-1, suggesting that Nesfatin-1 may play a role in VMH of diabetic rats through the adrenocorticotropic hormone receptor system. The occurrence and development of diabetic gastroparesis may be related to the effect of Nesfatin-1.For diabetic rats, electrical stimulation of hippocampal CA1 region can significantly promote gastric motility. Pre-injection of anti-Nesfatin-1 antibody in $\mathrm{VMH}$ partially blocked the stimulating effect of hippocampal CA1 on gastric motility. Therefore, we infer that hippocampal CA1 may be involved in the regulation of neuronal activity in $\mathrm{VMH}$. Fluorescence retrograde tracing and immunohistochemical staining also showed that there was Nesfatin-1 nerve fiber connection between hippocampal CA1 and VMH.

It has been reported that the expression of Nesfatin-1 in PVN of rats decreased significantly after fasting. The effects of Nesfatin-1 injection on different nuclei were different, and nighttime feeding decreased from 1 to 4 hours after PVN injection ${ }^{[9]}$. The effect of injecting LHA is similar to that of injecting PVN, but a higher dose is required. However, the effect of Nesfatin-1 injection in VMH on feeding has not been reported.Nesfatin-1 injected into lateral ventricle could inhibit gastric emptying and gastrointestinal activity in mice and rats ${ }^{[2,5,10]}$.

$\mathrm{VMH}$ has been the focus of research in the regulation of feeding behavior and body weight.Studies have reported that $\mathrm{VMH}$ lesions can lead to increased intake and obesity, so $\mathrm{VMH}$ is also known as the "satiety center" of the brain ${ }^{[8]}$. The rats with partial VMH injury may show excessive weight gain, but the effect of direct VMH injury on obesity is more obvious.Some scholars say that bilateral VMH injuries often lead to obesity ${ }^{[11]}$. Other studies have shown that the rate of overeating and weight gain in rats with bilateral VMH injury is significantly increased, and weight gain is related to the degree of VMH injury ${ }^{[12]}$. Later studies also found a high correlation between the size of $\mathrm{VMH}$ injury area and the weight gain after injury ${ }^{[13]}$.

NUCB2/Nesfatin-1 immunoreactive neurons can co-express with several other peptides and neurotransmitters to regulate food intake, including melanocyte concentrating hormone $(\mathrm{MCH})$, promelanocyte corticotropin (POMC), alpha-melanocyte stimulating hormone ( $\alpha-\mathrm{MSH})$, adrenocorticotropin

releasing hormone $(\mathrm{CRF})^{[5,14]}$.Nesfatin-1 can coexist with the neurotransmitters mentioned above. $\mathrm{CRF}$ receptor signaling system can also inhibit feeding ${ }^{[15,16]}$. Studies have shown that CRF signaling system may participate in Nesfatin-1-induced inhibition of feeding. It has been reported that injection of nesfatin-1 into the third ventricle increases the content of CRF in $\mathrm{PVN}^{[17]}$.Nesfatin-1 was injected into PVN to enhance the activity of CRF neurons ${ }^{[18]}$. These data strongly demonstrate that Nesfatin-1 can activate the hypothalamic-pituitary-adrenal axis through the release of CRF in PVN. In addition, pharmacological studies showed that lateral ventricle injection of CRF2 selective receptor antagonist astressin2-B could eliminate the effect of CRF2 receptor, and the third ventricle injection of CRF1/CRF2 antagonist $\alpha$-helicalCRF9-41 could partially block the anorexia effect of nesfatin- $1^{[19]}$. This study showed that astressin-B, a non-selective receptor antagonist of CRF, partially blocked the effect of nesfatin-1 on gastric motility in rats with VMH, suggesting that Nesfatin-1 and CRF signaling systems may interact and participate in the regulation of gastrointestinal function.

In conclusion, this study demonstrates that Nesfatin-1 injected by $\mathrm{VMH}$ can regulate gastric motility in diabetic rats, which may be related to the CRF system, and the hippocampal CA1 region is involved in the 
regulation of gastric motility by nesfatin-1 in VMH.This study provides a theoretical basis and therapeutic target for the diagnosis and treatment of diabetic gastrointestinal dysfunction-related diseases.

\section{References}

1. Cao X, Zhou X, Cao Y, et al. Expression of NUCB2/Nesfatin-1 in the taste buds of rats [J]. Endocr J, 2016, 63: 37-45.

2. Goebel-Stengel M, Wang LX, Stengel A, et al. Localization of Nesfatin-1 neurones in the mouse brain and functional implication [J]. Brain Res, 2011, 1396C: 20-34.

3. Atsuchi K, Asakawa A, Ushikai $\mathrm{M}$, et al. Centrally administered NUCB2/Nesfatin-1 inhibits feeding behaviour and gastroduodenal motility in mice [J]. NeuroReport, 2010, 21:1008-1011.

4. Ramesh N, Gawli K, Pasupulleti V, et al. Metabolic and Cardiovascular Actions of Nesfatin-1: Implications in Health and Disease [J]. Curr Pharm Des, 2017, [Epub ahead of print].

5. Gao S, Guo F, Sun X, et al. The Inhibitory Effects of Nesfatin-1 in Ventromedial Hypothalamus on Gastric Function and Its Regulation by Nucleus Accumbens [J]. Front Physiol. 2017, 7:634-644.

6. Schwartz MW, Woods SC, Porte Jr D, et al. Central nervous system control of food intake [J]. Nature, 2000, 404:661-671.

7. $\mathrm{Xu} \mathrm{L}$, Sun X, Depoortere I, et al. Effect of motilin on the discharge of rat hippocampal neurons responding to gastric distension and its potential mechanism [J]. Peptides, 2008, 29:585-592.

8. Schwartz MW, Woods SC, Porte Jr D, et al. Central nervous system control of food intake [J]. Nature, 2000, 404:661-671.

9. Goebel M, Stengel A, Wang L, et al Central Nesfatin-1 reduces the nocturnal food intake in mice by reducing meal size and increasing inter-meal intervals [J]. Peptides, 2011, 32:36-43.

10. Atsuchi K, Asakawa A, Ushikai $\mathrm{M}$, et al. Centrally administered Nesfatin-1 inhibits feeding behaviour and gastrodunal motility in mice [J]. Neuroreport, 2010, 21:1008-1011.

11. Liu Y, Gold MS. Human functional magnetic resonance imaging of eating and satiety in eating disorders and obesity [J]. Psychiatr Ann, 2003, 33:127-32.

12. Matsuda M, Liu Y, Mahankali S, et al. Altered hypothalamic response to oral glucose intake in obese humans [J]. Diabetes, 1999, 48: 1801-1806.

13. Routh VH. Glucosensing neurons in the ventromedial hypothalamic nucleus (VMN) and hypoglycemia-associated autonomic failure (HAAF) [J]. Diabetes Metab Res Rev, 2003, 19: 348-356.

14. Okere B, Xu L, Roubos EW, et al. Restraint stress alters the secretory activity of neurons co-expressing urocortin-1, cocaine-and amphetamine-regulated transcript peptide and Nesfatin-1 in the mouse Edinger-Westphal nucleus [J]. Brain Res, 2010, 1317C: 92-99.

15. Zorrilla EP, Tachy, Koob GF. Nibbling at CRF receptor control of feeding and gastrocolonic motility [J]. Trends PharmacolSci, 2003, 24:421-427.

16. Tabarin A, Diz-Chaves Y, Consoli D, et al. Role of the corticotropin-releasing factor receptor type 2 in the control of food intake in mice: a mean pattern analysis [J]. Eur J Neurosci, 2007, 26:2303-2314.

17. Gotoh K, Masaki T, Chiba S, et al. Nesfatin-1, corticotropin releasing hormone, thyrotropin-releasing hormone, and neuronal histamine interaction in the hypothalamus to regulate feeding behavior [J]. J Neurochem, 2013, 124:90-99.

18. Price CJ, Hoyda TD, Samson WK, et al. Nesfatin-1 influences the excitability of paraventricular nucleus neurons [J]. J Neuroendocrinol, 2008, 20:245-250.

19. Stengel A, Goebel M, Wang L, et al. Central Nesfatin-1 reduces dark-phase food intake and gastric emptying in rats: differential role of corticotropin-releasing factor 2 receptor [J]. Endocrinology, 2009, 150:4911-4919. 